\title{
Nootropics in Postmodernity:
}

\author{
What the Philosophy of Gilles Deleuze Can Tell Us About the \\ Relationship Between Smart Drugs, Authenticity, Control and \\ Fairness
}

\begin{abstract}
Sean Blanchet and Sean Devine*
Pharmaceutical products aimed at enhancing cognitive performance - smart drugs, nootropics, etc - have garnered considerable attention. Many ethical questions accompany their growth in popularity. Three of these questions will be addressed in this paper: Do nootropics preclude authenticity? Are nootropics a good thing for society? And, is it fair to use nootropics? We argue that these questions can be answered by appealing to Gilles Deleuze and Felix Guattari's concepts of individuation, Society of Control, and dividuation. On the one hand, drawing on the concept of extrinsic individuation, we posit that nootropics have the potential to expand horizons, disrupt traditional human-object interactions, and allow users to experience their surroundings in novel, more authentic, ways. On the other, it also risks concentrating success in the hands of a minority of individuals and reinforce the normative power of the Society of Control. Ultimately, we suggest that whether it is fair, indeed ethical, to use nootropics depends on the control the user has over herself and her cognition within the Society of Control.
\end{abstract}

\section{Introduction}

From early weaponry to modern vaccines, the pursuit of progress through technological means can be found in nearly every human society. Today, we see this pursuit manifest in the form of cognitive enhancement. From synthetic drugs to music and exercises, cognitive enhancement can take many shapes. That being said, in a world in which our success often depends on our cognitive skills, fast-acting nootropic drugs have become increasingly popular. But, a number of important ethical questions accompany this increase in popularity. In this short article, we want to tackle three questions for a society in which nootropics are becoming increasingly commonplace, with a particular focus on synthetic nootropic drugs. ${ }^{1}$ First, does nootropic use preclude authentic expression? Nootropics change the brain. If their use becomes commonplace, it would be important to discern to what extent behaviour is a result of individual volition or the drugs' influence. Moreover, we want to consider the possibility that these drugs might in fact enhance authentic expression rather than limit it. Second, are nootropics a good thing for society at large? On the one hand, a smarter society might be a better society. The more people that take nootropics, the higher the cognitive performance of the average individual; the more difficult problems solved, the better off society is. On the other hand, the prioritisation of a certain set of cognitive abilities (eg, spatial reasoning, fluid abilities, etc) might come with the discouragement of others (eg, creativity, awkwardness, quirkiness, etc). At its most extreme, this approach risks minimising cognitive diversity and lead to a homogeneous mass of people unable to think outside the confines of what has been prescribed for them. Third, is it fair to use nootropics? As with any commodity that is bought

DOI: 10.21552/delphi/2018/1/10

* Sean Blanchet and Sean Devine are independent authors from Montréal, Canada. For correspondence: <Sean.blanchet.mail@gmail .com $>$ and $<$ seandamiandevine@gmail.com $>$ respectively. The order of authors is alphabetical; both authors contributed equally.

1 This is not to say that natural nootropics do not play an important role in society however, nor that they do not come with ethical baggage themselves. Indeed, this is an area that merits (requires) more research, but falls outside of the scope of this paper. 
and sold, there is a risk of inequality. For nootropics, this inequality risks not only to be economic, but also cognitive: a society in which the rich have the means to consistently outperform the poor.

Ethicists have proposed many different answers to these questions. ${ }^{2}$ But, they have mainly treated the use of nootropics as either an individual choice or an issue for society at large. In actuality however, the two are not separate. The individual is shaped by her surroundings, and her surroundings are shaped by her. This dynamic interplay between the individual and her environment can be best understood through the works of French philosophers Gilles Deleuze and Felix Guattari. For Deleuze and Guattari, the relationship between the individual and society is complicated. On the one hand, they recognise the undeniable effect that Societies of Control have on the individual. Building on the work of Michel Foucault, they highlight how sociotechnological advances in society can contribute to a life of servitude and indeed even mold an individual's subjectivity through a process known as dividuation. ${ }^{3}$ On the other hand, the pair are optimistic about the individual's capacity to challenge and transcend the constricting hold of the state: to live as a nomad, as they put it. ${ }^{4}$ Importantly, doing so requires a deep critique and expansion of one's perception away from the limits imposed on it by the state. As Genn-Bash has pointed out however, for Deleuze, the individual's relationship to the status quo, to the state, might critically depend upon the use of external substances; that is, it might depend on the use of drugs. ${ }^{5}$ As such, we suggest that nootropics have the potential to simultaneously reinforce and challenge the Society of Control.

With this in mind, we argue that the philosophy of Deleuze and Guattari can be used to provide nuanced answers to our three questions: does nootropic use preclude authentic expression? Are nootrop-

2 For a review and an example of some responses, see Arthur Saniotis, 'Remaking Homo: ethical issues on future human enhancement' (2013), Ethics in Science and Environmental Policies <https://www.int-res.com/articles/esep2013/13/e013p015.pdf> accessed 23 August 2018

3 Gilles Deleuze, Postscripts on the Societies of Control (1992)

4 Gilles Deleuze \& Félix Guattari, A Thousand Plateaus (University of Minnesota Press, 1987) 380

5 Oli Genn-Bash, 'Gilles Deleuze and Psychedelic Thought as Resistance' in David Luke and Dave King (eds), Neurotransmissions: Essays on Psychedelics from Breaking Convention (Strange Attractor Press 2015) ics a good thing for society? And is it fair to use nootropics? To answer these questions, we will appeal to Deleuze and Guattari's concepts of individuation, Society of Control, and dividuation. As nootropic use becomes more commonplace, it is crucial to consider their ethical implications within the postmodern condition. Given Deleuze and Guattari's important influence on the postmodern condition, we believe their work can provide valuable insight into the ethical implications of nootropic use today.

\section{Does Nootropic Use Preclude Authentic Expression?}

One of Deleuze and Guattari's key insights is that neither the society nor the individual exist in isolation; societies are formed by individuals and individuals are molded by societal influence. This notion is captured most succinctly in their process of individuation. To understand what is meant by individuation, we must first keep in mind that every individual is subject to this process. One cannot take on a role in an institution, a job, or a household without being considered individuated or becoming individuated. Second, we need to avoid our desire to define individuation (as with any of Deleuze and Guattari's concepts) as strictly good or bad. Instead, we should observe whether the elements that mold this process allow for a continuous, dynamic, (nomadic, if you will) development or if they emerge from a static environment where the result is final and considered unchangeable. In other words, whether it is a process of intrinsic individuation, where the properties of the individual are fixed and innate, or extrinsic individuation, where the individual adapts, molds and changes according to the changing environment. This difference is best captured in this section of $A$ Thousand Plateaus:

Chess pieces are coded; they have an internal nature and intrinsic properties from which their movements, situations, and confrontations derive. They have qualities; a knight remains a knight, a pawn a pawn, a bishop a bishop. Each is like a subject of the statement endowed with a relative power, and these relative powers combine in a subject of enunciation, that is, the chess player or the game's form of interiority. Go pieces, in contrast, are pellets, disks, simple arithmetic units, and have only an anonymous, collective, or third-person 
function: 'It' makes a move. 'It' could be a man, a woman, a louse, an elephant. Go pieces are elements of a nonsubjectified machine assemblage with no intrinsic properties, only situational ones. Thus the relations are very different in the two cases. Within their milieu of interiority, chess pieces entertain biunivocal relations with one another, and with the adversary's pieces: their functioning is structural. On the other hand, a Go piece has only a milieu of exteriority, or extrinsic relations with nebulas or constellations as bordering, encircling, shattering. All by itself, a Go piece can destroy an entire constellation synchronically; a chess piece cannot. ${ }^{6}$

Within the context of nootropics, we speak of being extrinsically individuated. That is, characteristics that were once outside of the eyes of society, systems of technology, and even ourselves are now being evaluated and measured, ultimately contributing to new possibilities and the lifting of restrictions. New properties are evaluated and come to define our place and role on the chess board. New rules, new opponents, and new opportunities on the board re-define your options as a Go piece. Put simply, the ability to temporarily modify our brains in order to increase performance tears down boundaries. Fixed elements, those that were once largely unalterable by the individual, such as brain chemistry and cognitive capacity, become variable. Those who were discredited or singled out, considered unable to attain certain positions due to certain particularities, could find ways to experiment with their capacities. That is, they could subvert or compensate for what might currently be considered a 'weakness' by further exploring their own particularities with the help of nootropics. In this context, nootropics allow for greater extrinsic individuation. They give us the opportunity to express our peculiarities, our personal traits, and our authentic selves, in new and interesting ways.

\section{Are Nootropics a Good Thing for Society?}

To answer this question, we can appeal to Deleuze and Guattari's notion of the Society of Control. The Society of Control is different than Societies of Discipline (a concept popularised by Michel Foucault). In Societies of Discipline, control is maintained through a rigid combination of systems of technology and rules forming an all-seeing and all-powerful authority (known as a panopticon). In contrast, Societies of Control do not forcefully exert their authority on their subjects. Rather, they guide and evaluate their behaviour. To be clear, Societies of Control are not less oppressive nor more progressive than Societies of Discipline. Control is achieved and maintained through the use of the corporate structure and an inherent culture of competition. By pitting one against the other in a contest of productivity, in a maze of meetings and group sessions (often done in the name of 'team building', even though the individually varying nature of salary does not encourages you to partake in cooperation), individuals come to be controlled by a master that they can't name. Instead of a progression, the shift from Society of Discipline to one of Control marks a transition from a binary model of control (conformity vs nonconformity, productive vs unproductive, etc) to a numerical model, in which you are assigned a specific value (eg, individually varying salaries) and different institutions, corporations or otherwise, determine your possible use. As Deleuze puts it in Postscripts on the Societies of Control: 'We no longer find ourselves with the mass/individual pair. Individuals have become dividuals, and masses, samples, data, markets, "banks"." The process of dividuation consists of the internal division of entities into measurable and adjustable parameters, in a similar fashion, to say, a pig or cow that is divided into multiple sectors (cuts of meat) and accompanied by a set of instructions giving detailed information on how one adjusts the preparation of said meat depending from on which sector it comes from.

Importantly, these societies don't gain control in some deterministic way. Control is reinforced by sociotechnological change. As technology comes to occupy a predominant role within society, it comes to shape our expectations of each other. Whether someone is good or bad, successful or unsuccessful, valuable or dispensable, is inherently linked to what tools we use to measure them. This way, nootropics risk to reinforce the Society of Control. By breaking up cog-

6 Gilles Deleuze \& Félix Guattari, A Thousand Plateaus (University of Minnesota Press 1987) 353

7 Michel Foucault, Discipline and Punish: The Birth of a Prison (Gallimard 1975)

8 Gilles Deleuze, Postscripts on the Societies of Control (1992) 5 
nitive abilities and ways of thinking into sectors, by dividuating people, control is seized over yet another aspect of our lives. A numerical code is assigned to our abilities and those who fail to meet a certain cut-off face persecution within the society. Of course, it might be said that this is already happening. The risk with nootropics is that we provide a material commodity with which this cutting-up is done. The brain becomes like any other piece of meat: divided, scaled, and sold for social value, all while the butcher, the Society of Control, profits and becomes more powerful.

\section{Is It Fair to Use Nootropics?}

With regards to fairness, the questions of control and authenticity might seem trivial. If using nootropics is fair, we might be less concerned with control and perhaps more willing to use them for reasons of authenticity. If they are unfair, the opposite might be true. As we have seen thus far with Deleuze and Guattari though, these questions are seldom separate and rarely so black and white. As we've seen, nootropics contain an emancipatory potential. They can extrinsically individuate and promote authentic expression. Through continued, widespread use, they offer the opportunity for individuals to challenge the Society of Control and assert power. Further, this process of autonomous assertion has the potential to guide the development of future nootropics away from the desires of the society and towards a more open, community-driven, means for improvement and authenticity ${ }^{9}$. In this way, nootropics can be very fair. However, we must recognise that currently nootropics are both funded by and made for individual who profit from a world that is heavily regulated and productivity-oriented; they are made by and for businesses. And as with any business, the end goal is profit. As such, within the corporate structure, a place where competition and productivity reign, nootropics are less of a tool of self-exploration and self-expression, but more a means to increase productivity and diminish costs. Were nootropics to become commonplace, this could manifest both from the top and the bottom. In a top-down model, coorporations could impose regulations that directly or

9 We are grateful to the reviewers for pointing this out. indirectly select for certain capacities. For instance, a tech startup might require all employees to have two different versions of an app ready for Friday. To complete this task, employees would have to work long-nights and expend great cognitive effort. Of course, this type of work is entirely feasible when nootropics are available. Indeed, it might only be possible using nootropics. In this way, nootropics are still being used to enhance certain qualities within an individual, but we are far from the extrinsic, authentic, individuation we saw earlier. Rather, the Society of Control, through the corporate structure, tightens its grips as it dividuates the individual, transforming her particularities, her uniqueness, into a value. Should that value fall below some threshold, the individual risks exclusion or even persecution. This continued exclusion of undesirable capacities leads to a more homogeneous, conforming, and controllable mass. In a bottom-up model, we might see employees using nootropics competitively to get a legup on their coworkers. Since nootropics are not free, this competitive drive could reify pre-existing class divisions. Those with the means to afford cognitive enhancement can consistently outperform those who don't and as a result they accumulate even more wealth (ie, they get the promotion, they get the bonus, etc). In this case, unfairness begets unfairness in both models.

\section{Conclusions}

We argue that the ethical status of nootropic use is not inherent in the technology, but depends critically on the relationships it forms with society and the individual. Deleuze and Guattari show us above all that cognitive enhancement has both the potential for emancipation and exploitation. On the one hand, motivated by a desire to transcend limitations and explore one's self, nootropic use can extrinsically individuate, allowing us to transform the way we experience and interact with our world. At its most powerful, it can be used as tool to promote authentic individuality and challenge the status quo. On the other hand, it can be used to dividuate, enforce normative behaviour, and deepen the Society of Control's influence on our lives. Nootropics can be used to promote a certain set of behaviours deemed valuable by the society at the expense of those deemed undesirable. At the practical level, this reinforcement of so- 
ciety's control is done by corporations that have the material power to effectively enforce (explicit and implicit) rules that do this dividuating. As such, whether using nootropics is fair, whether it is ethical in the long-run, will depend on the dynamic interplay between the individual and the society and wherein the power of the user lies. The transcendent potential of nootropics can only be realised if it exists in an open plane where individual potential can be explored, expanded, and ultimately enhanced.

Though the majority of this paper has been theoretical, we hope to conclude by remarking that many of these ideas are not just speculative. Cognitive enhancers are already being marketed across silicon valley as miracle drugs ${ }^{10}$ and prescription drugs like
Adderall are being abused in schools to help students study every day. ${ }^{11}$ Technology does not exist in a vacuum; it is deeply connected to the social millieu in which it finds itself. In the postmodern condition, these technologies determine our lives just as we determine their use.

10 Jillian D'Onfro, 'Marissa Mayer and Mark Pincus invested in a startup that makes 'brain drugs' and chewable coffee' (Business Insider, October 13, 2015) <https://www.businessinsider.com/ nootrobox-nootropics-startup-raises-seed-round-of-funding-2015 $-10>$ accessed 12 September, 2018

11 Christian J. Teter, Sean Esteban McCabe, Kristy LaGrange, James A. Cranford, and Carol J. Boyd, 'Illicit Use of Specific Prescription Stimulants Among College Students: Prevalence, Motives, and Routes of Administration' (2006) Pharmacotherapy 